\title{
Another step towards biomolecular microscopy
}

\author{
Atomic Force Microscopy for Biologists \\ by V. J. Morris, A. R. Kirby and A. P. Gunning \\ Imperial College Press · January 2000 \\ Hardback $£ 32.50 / \$ 51$
}

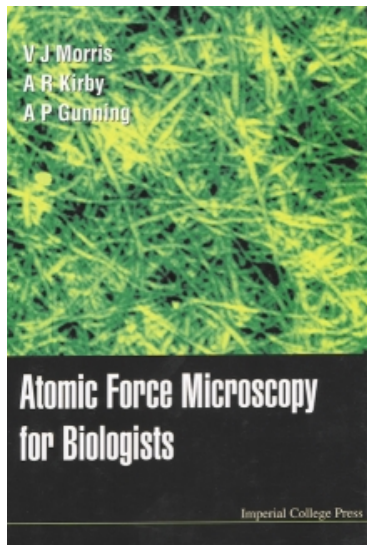

dimensions by the piezoelectric transducer. Mapping the surface topography of specimens in this manner generated such startling atomic images that the invention of the STM was recognized with the 1986 Nobel Prize for physics. The atomic-force microscope (AFM) uses a similar scanning mechanism, but generates image contrast differently - by measuring the deflection of a micro-machined silicon-oxynitride cantilever with an integral tip. Interactions with the specimen deflect the cantilever from its equilibrium position, which can be monitored by a laser beam reflected from the back of the cantilever into a positionsensitive detector. In its simplest form, AFM measures the surface topography by monitoring the cantilever deflection as the specimen is raster-scanned. Thus, any variant of AFM that alters the tip-specimen interaction (charge, chemical bonds, etc.) can be used to generate useful contrast from an SPM. In principle, SPM S should provide resolution of biological specimens that is comparable to or better than that obtainable with electron microscopes, but in more biologically relevant imaging environments such as physiological buffers.

Atomic Force Microscopy for Biologists fills an important niche by providing an introduction to AFM that will be understandable to a wide range of scientists. The book offers an overview of the key components common to all SPM s, explaining the theory of their operation as well as the limitations in their performance. In a little more than three hundred pages, the authors are thus able to offer the reader an overview of what can and, more importantly, what cannot be deduced from AFM data. The book presents the techniques and the special concerns relating to obtaining AFM data from specimens ranging from proteins and membranes to a variety of entire cells. The book is far too brief to be considered a laboratory guide, but this is a strength, not a weakness. The recipes and details expected in a lab manual would make a book that is very unlikely to reach a broad audience. Much as computer manuals seem to make the best sense to those who al ready know the answer to a question, lab manuals usually serve best to inform those al ready familiar with at least the basics of an approach. Atomic Force M icroscopy for Biologists takes care to offer enough detail to be substantial, but avoids the temptation to heap too many details on the reader. In the last few pages it offers a brief mention of other SPM s that are related to AFM. These few words inform the reader of emerging technologies and give a brief introduction to their mechanisms of operation. I recommend Atomic Force M icroscopy for Biologists because the reader is offered an introduction of sufficient brevity to actually be read and of sufficient depth to help in defining the potential uses of SPM $s$ in his or her own research.

Scott Fraser is at Caltech, Pasadena,

California 91125, USA

e-mail: sefraser@caltech.edu

\section{Other microscopy books}

Paperback

Light and Electron Microscopy by Elizabeth M. Slayter and Henry S. Slayter

Cambridge University Press, $£ 23 / \$ 15$

Methods in Cell Biology: Cell

Biological applications of confocal microscopy vol. 38

edited by Brian Matsumoto

Academic Press, $£ 43 / \$ 62$

\section{Essays of the History of the Light} Microscope

by Gerard L'Estrange Turner Senecio, $f 14.95 / \$ 20$

Maintaining and Monitoring the Transmission Electron Microscope by S. K. Chapman

Bios, $£ 9.95 / \$ 15$ 